\title{
Nail-Patella Syndrome: A Classic Case
}

\author{
Sarita Sanke ${ }^{1}$, Taru Garg ${ }^{1}$, Rubina Jassi ${ }^{1}$, Apoorva Maheshwari ${ }^{1}$
}

1 Department of Dermatology, Lady Hardinge Medical College \& Associated Hospitals, New Delhi, India

Key words: nail patella, elbow pterygium, iliac horns, dermoscopy

Citation: Sanke S, Garg T, Jassi R, Maheshwari A. Nail-patella syndrome: a classic case. Dermatol Pract Concept. 2019;9(4):271. DOI: https://doi.org/10.5826/dpc.0904a04

Accepted: July 3, 2019; Published: October 31, 2019

Copyright: $@ 2019$ Sanke et al. This is an open-access article distributed under the terms of the Creative Commons Attribution License, which permits unrestricted use, distribution, and reproduction in any medium, provided the original author and source are credited.

Funding: None.

Competing interests: The authors have no conflicts of interest to disclose.

Authorship: All authors have contributed significantly to this publication.

Corresponding author: Rubina Jassi, MD, DNB, MRCP (SCE), Department of Dermatology, Lady Hardinge Medical College, Shaheed Bhagat Singh Marg, New Delhi 110001, India. Email: jassi.rubina@gmail.com

\section{Case Presentation}

A 41/2-year-old boy presented with a delayed appearance of all fingernails and near absence of both thumbnails, present since birth, along with incomplete extension of the bilateral elbows. On examination, the bilateral thumbnails were hypoplastic and the index fingers of both hands showed tenting with triangular lunulae. Flexion deformity was noted at the bilateral elbows. A roentgenogram of the pelvis showed bilateral iliac horns, while that of both knees showed absent patella. The rest of the examination was within normal limits. On the basis of the presence of bilateral iliac horns, triangular lunulae, webbing of elbow, and an absent patella (Figure 1, A-D), a diagnosis of nail-patella syndrome was made.

\section{Teaching Point}

Nail-patella syndrome presents with a classic tetrad of triangular lunulae, elbow pterygium, absent patellae, and posterior iliac horns. Dermoscopy aids in better visualization of the triangular lunulae.

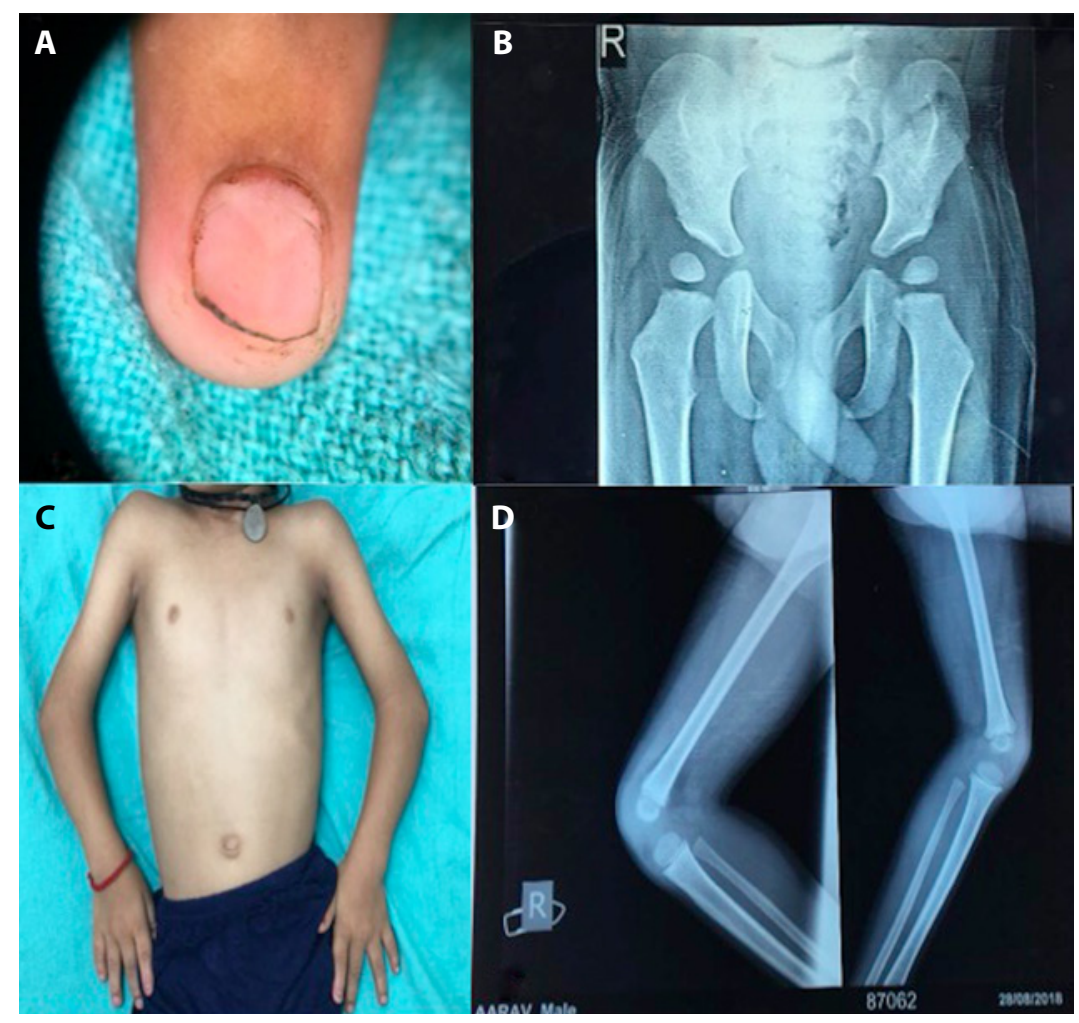

Figure 1. Nail-patella syndrome collage showing (A) triangular lunula on dermoscopy, (B) bilateral posterior iliac horns, (C) bilateral elbow pterygium, and (D) absent patella. [Copyright: (C2019 Sanke et al.] 